\title{
Sub-100 femtosecond pulses from a SESAM modelocked thin disk laser
}

\author{
C.J. Saraceno • O.H. Heckl • C.R.E. Baer • C. Schriber • \\ M. Golling • K. Beil · C. Kränkel · T. Südmeyer • \\ G. Huber · U. Keller
}

Received: 9 January 2012 / Published online: 26 January 2012

(c) The Author(s) 2012. This article is published with open access at Springerlink.com

\begin{abstract}
We present the first passively modelocked thin disk laser (TDL) with sub-100-fs pulse duration using the broadband sesquioxide gain material $\mathrm{Yb}: \mathrm{LuScO}_{3}$ and an optimized SEmiconductor Saturable Absorber Mirror (SESAM). In this proof-of-principle experiment, we obtained $5.1 \mathrm{~W}$ of average power at a repetition rate of $77.5 \mathrm{MHz}$ and a pulse duration of $96 \mathrm{fs}$. We carefully explored and optimized the different parameters on the soliton pulse formation process for the generation of short pulses. In particular, SESAMs combining fast recovery time, high modulation depth and low nonsaturable losses proved crucial to achieve this result even though they are expected to only play a minor role in soliton modelocking. To our knowledge, these are the shortest pulses ever obtained with a modelocked TDL, reaching for the first time the sub-100-fs milestone. This result opens the door to sub-100-fs oscillators with substantially higher power levels in the near future.
\end{abstract}

\section{Introduction}

To date passively modelocked thin disk lasers (TDLs) [1] using SEmiconductor Saturable Absorber Mirrors (SESAMs)

C.J. Saraceno $(\bowtie) \cdot$ O.H. Heckl · C.R.E. Baer · C. Schriber ·

M. Golling · C. Kränkel · T. Südmeyer · U. Keller

Department of Physics, Institute for Quantum Electronics,

ETH Zurich, 8093 Zurich, Switzerland

e-mail: saraceno@phys.ethz.ch

K. Beil · C. Kränkel · G. Huber

Institute of Laser-Physics, University of Hamburg,

Luruper Chaussee 149, 22761 Hamburg, Germany

Present address:

O.H. Heckl · T. Südmeyer

Department of Physics, University of Neuchâtel, 2000 Neuchâtel,

Switzerland
[2] enable the highest average powers and pulse energies of any femtosecond oscillator technology [3]. The thin gain medium of only a few $100 \mu \mathrm{m}$ allows for efficient heat removal and provides excellent linear and nonlinear optical properties. A record high average output power of $141 \mathrm{~W}$ with a pulse duration of $738 \mathrm{fs}$ has been demonstrated with the novel sesquioxide gain material $\mathrm{Yb}: \mathrm{Lu}_{2} \mathrm{O}_{3}$ [4], whereas a pulse energy $>30 \mu \mathrm{J}$ has been demonstrated in 1-ps pulses using the well-established gain material Yb:YAG [5]. In principle, the output power of modelocked TDLs can be scaled up by increasing the beam diameters on both the thin disk gain medium and the SESAM, without detrimental nonlinear effects. TDLs are therefore excellent candidates for applications that require energetic femtosecond pulses from table-top sources operating at $\mathrm{MHz}$ repetition rates $[6,7]$.

To date, sub-100-fs pulses directly from a TDL have not been achieved [8]. Therefore, external pulse compression of TDLs was applied based on different techniques such as passive spectral broadening using self-phase modulation (SPM) [9], active spectral broadening in fiber amplifiers [10], and compression in gas-filled hollow core fibers [11]. Although impressive performance has been reached with these systems, they usually suffer from a reduced temporal quality of the pulses and an increased complexity compared to a simple SESAM modelocked laser oscillator. SESAM modelocked thin disk lasers operate in the soliton modelocking regime [12], supporting transformlimited $\operatorname{sech}^{2}$-shaped pulses directly from an oscillator with a footprint similar to that of a low-power oscillator.

A strong effort in novel $\mathrm{Yb}$-doped laser material development $[13,14]$ is motivated by sub-100-fs pulse generation from modelocked TDLs. This requires not only a broad emission bandwidth $[15,16]$ but also excellent optical and mechanical properties for manufacturing and mounting thin disks with a crystal thickness in the $100-\mu \mathrm{m}$ range. 


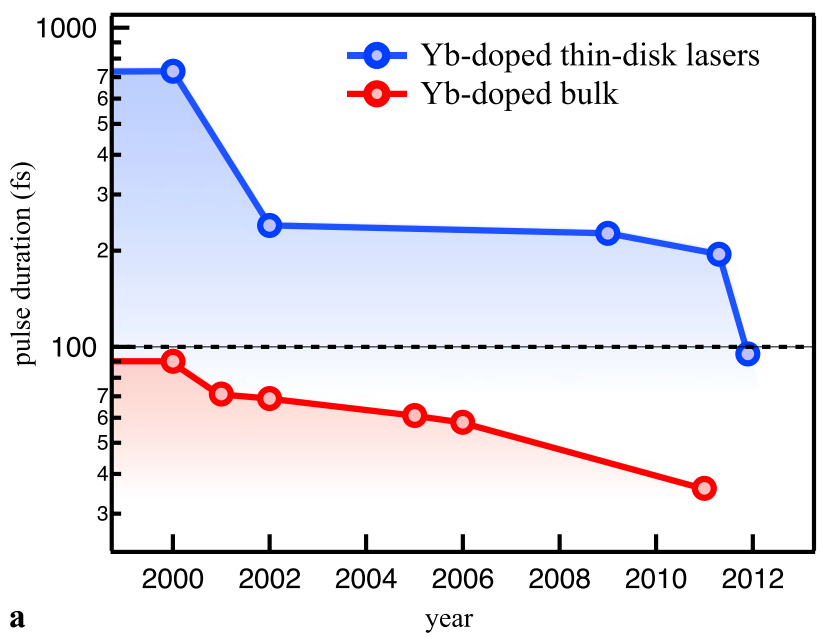

Fig. 1 Overview of the pulse duration of modelocked thin disk lasers: (a) Evolution of the minimum pulse duration of modelocked thin disk lasers compared to other Yb-doped oscillators, showing the recent progress in terms of pulse duration reaching for the first time the

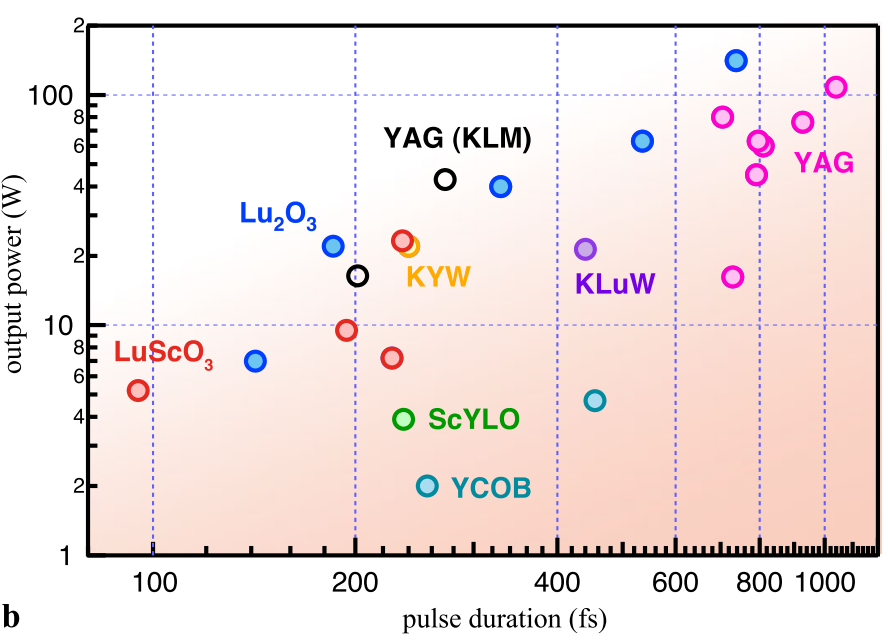

sub-100-fs regime. (b) Average power of SESAM modelocked thin disk lasers versus pulse duration. The gradient illustrates the current research effort to extend short pulse durations into the multi-100-W regime
Fig. 2 Schematic of the $\mathrm{Yb}: \mathrm{LuScO}_{3}$ modelocked thin disk laser cavity that allowed the demonstration of sub-100-fs pulses

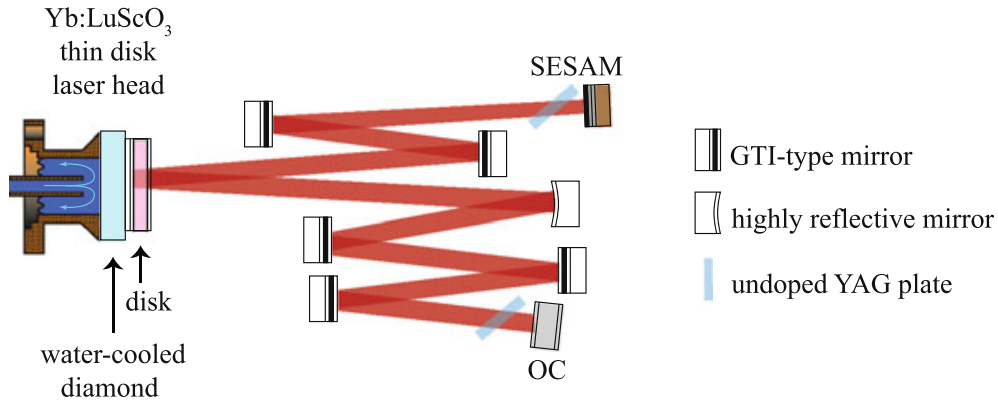

Since the first demonstration of modelocked TDLs in the year 2000 [17], seven different Yb-doped materials have been investigated in modelocked operation in the thin disk geometry (Fig. 1). For many years, Yb:KYW held the record in terms of pulse duration with $240 \mathrm{fs}$ at an average power of $22 \mathrm{~W}$ [18]. However, further power scaling was hindered by the anisotropic thermal properties of this material. Very recently, the first Kerr lens modelocked (KLM) [19] TDL was demonstrated using Yb:YAG generating $17 \mathrm{~W}$ average power with pulse duration of $200 \mathrm{fs}$ [20]. Recent progress in the growth of high quality sesquioxide materials has attracted great attention [21, 22]. $\mathrm{Yb}: \mathrm{Lu}_{2} \mathrm{O}_{3}$ already holds the record in terms of average power from a passively modelocked oscillator [4] and very recently, 142 fs pulses were reported from this material exploiting $>70 \%$ of the available $12 \mathrm{~nm}$ emission bandwidth [23]. This shows the great potential of this material also in terms of short pulse generation. Even larger emission bandwidths can be reached by growing mixed sesquioxide materials $[24,25]$ such as the isotropic material $\mathrm{Yb}: \mathrm{LuScO}_{3}$ with $22 \mathrm{~nm}$ of emission bandwidth. This material has already proved its great potential for short pulse generation with the recent demonstration of
195 fs [26]. Here, we used the same gain material but optimized key components for broadband modelocking. In this way, we were able to demonstrate $5.1 \mathrm{~W}$ of average power at a repetition rate of $77.5 \mathrm{MHz}$ and a pulse duration of $96 \mathrm{fs}$, reaching the sub-100-fs regime for the first time. In the near future, power scaling will allow to reach the multi- $100 \mathrm{~W}$ level at these short pulse durations.

\section{Experimental setup}

The laser setup is shown in Fig. 2. We used a 200- $\mu$ m thick $\mathrm{Yb}: \mathrm{LuScO}_{3}$ thin disk mounted on a 1.4-mm thick diamond which was water-cooled directly through the back surface for efficient heat removal. Efficient pump absorption in the narrow zero-phonon line of $\mathrm{Yb}: \mathrm{LuScO}_{3}$ was achieved with a volume Bragg grating (VBG) stabilized pump diode [27] emitting at a central wavelength of $976 \mathrm{~nm}$ with a narrow linewidth of $<0.5 \mathrm{~nm}$. The pump module was arranged for 24 pump passes through the disk, enabling a pump absorption of $>95 \%$. The pump spot diameter on the disk used throughout the experiment was $1.9 \mathrm{~mm}$. For stable soliton 
Fig. 3 Sub-100-fs thin disk laser. Autocorrelation trace and optical spectrum analyzer trace of the sub-100-fs pulses obtained at an average power of $5.1 \mathrm{~W}$

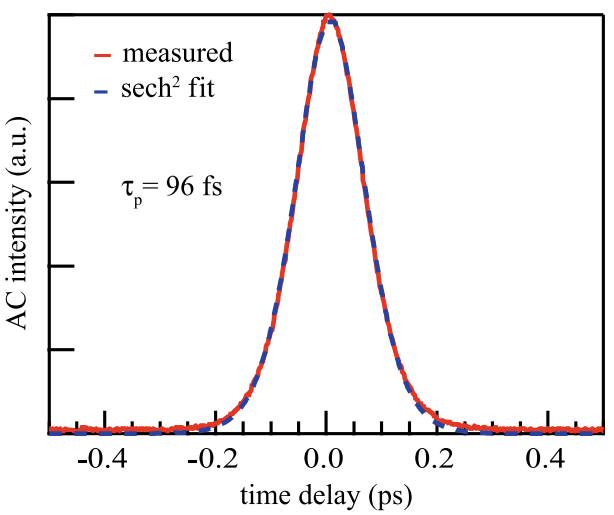

modelocking [15], we introduced five Gires-Tournois Interferometer (GTI) type mirrors that accounted for $2800 \mathrm{fs}^{2}$ of negative group delay dispersion (GDD) per roundtrip. Two uncoated YAG plates inserted at Brewster's angle with thicknesses of 5 and $7 \mathrm{~mm}$ maintain linear polarization and introduce self-phase modulation (SPM) to balance the negative GDD. Using only one plate would minimize small losses introduced at Brewster's angle. However, at the time of the experiment, the maximum thickness available was $7 \mathrm{~mm}$, therefore, two plates were introduced at the expense of a small loss in output power. We used an output coupler with $2.6 \%$ transmission and an uncoated SESAM with the following measured parameters [28]: saturation fluence $F_{\text {sat }}=36 \mu \mathrm{J} / \mathrm{cm}^{2}$, a modulation depth $\Delta R=3.3 \%$, nonsaturable losses $\Delta R_{\mathrm{ns}}=0.7 \%$, and a fast recovery time of $\tau_{1 / e}=1.9 \mathrm{ps}$.

\section{Modelocking results}

Stable modelocking was obtained with up to $5.1 \mathrm{~W}$ of average power with an optical-to-optical efficiency of $11 \%$. The pulse duration at the maximum power level was $96 \mathrm{fs}$ (Fig. 3). The laser operated at a repetition rate of $77.5 \mathrm{MHz}$. The pulses were close to the transform-limit of the spectrum with a time-bandwidth product of 0.33 (ideal $\operatorname{sech}^{2} 0.315$ ). These are, to our knowledge, the shortest pulses ever obtained from a modelocked TDL, reaching for the first time the sub-100-fs regime.

This experiment targeted an investigation of the limits in terms of pulse duration of soliton modelocked TDLs. According to theoretical predictions, the key element to achieve short pulses is the gain bandwidth of the material $[15,16]$. Although the SESAM parameters have only a small influence on the minimum achievable pulse duration, large modulation depths, and fast recovery times are beneficial to push the pulse duration to the limits. This was confirmed with our current result because key improvements in SESAM design resulted in sub-100-fs pulse durations for the first time. These new SESAMs combined $>3 \%$ modulation depth and an extraordinary fast recovery time of $\tau_{1 / \mathrm{e}}=1.9 \mathrm{ps}$. In our case, we used small laser mode areas on the gain medium and the absorber, which resulted in a moderate average output power but allowed for a relaxed cavity configuration [29] without Q-switching instabilities [30]. Future SESAMs with multiple quantum wells and dielectric topcoatings for higher saturation fluences and damage thresholds [31] are expected to reach higher power levels.

\section{Conclusion and outlook}

Our experimental results represent a first proof-of-principle demonstration of modelocked TDLs with sub-100-fs pulse duration. This was achieved with the broadband sesquioxide material $\mathrm{Yb}: \mathrm{LuScO}_{3}$ (22 nm of FWHM emission bandwidth) and optimized SESAM parameters. Although the output power level in this experiment is moderate, higher powers are feasible at these short pulse durations by using larger disks, increased mode areas on the gain medium and on the SESAM. Furthermore, designing SESAMs with similar fast recovery times and high modulation depths but larger saturation fluences, lower two-photon absorption effects and higher damage thresholds [31] will support further power scaling at these short pulse durations. We target power scaling of SESAM modelocked TDLs to the multi$100-\mathrm{W}$ regime with sub-100-fs pulse durations.

Acknowledgements We would like to acknowledge financial support by the Swiss National Science Foundation (SNF) and support from the FIRST cleanroom facilities of ETH Zurich for the SESAM fabrication. Christian Kränkel acknowledges financial support by the Joachim Herz Stiftung.

Open Access This article is distributed under the terms of the Creative Commons Attribution License which permits any use, distribution, and reproduction in any medium, provided the original author(s) and the source are credited.

\section{References}

1. A. Giesen, H. Hügel, A. Voss, K. Wittig, U. Brauch, H. Opower, Appl. Phys. B 58, 365 (1994) 
2. U. Keller, K.J. Weingarten, F.X. Kärtner, D. Kopf, B. Braun, I.D. Jung, R. Fluck, C. Hönninger, N. Matuschek, J. Aus der Au, IEEE J. Sel. Top. Quantum Electron. 2, 435 (1996)

3. U. Keller, Appl. Phys. B 100, 15 (2010)

4. C.R.E. Baer, C. Kränkel, C.J. Saraceno, O.H. Heckl, M. Golling, R. Peters, K. Petermann, T. Südmeyer, G. Huber, U. Keller, Opt. Lett. 35, 2302 (2010)

5. D. Bauer, F. Schättiger, J. Kleinbauer, D. Sutter, A. Killi, T. Dekorsy, Energies above $30 \mu \mathrm{J}$ and average power beyond $100 \mathrm{~W}$ directly from a mode-locked thin-disk oscillator, presented at the Advanced Solid-State Photonics, Istanbul, Turkey, 2011

6. T. Südmeyer, S.V. Marchese, S. Hashimoto, C.R.E. Baer, G. Gingras, B. Witzel, U. Keller, Nat. Photonics 2, 599 (2008)

7. U. Keller, IEEE Photonics J. 2, 3 (2010)

8. T. Südmeyer, C. Kränkel, C.R.E. Baer, O.H. Heckl, C.J. Saraceno, M. Golling, R. Peters, K. Petermann, G. Huber, U. Keller, Appl. Phys. B 97, 281 (2009)

9. T. Südmeyer, F. Brunner, E. Innerhofer, R. Paschotta, K. Furusawa, J.C. Baggett, T.M. Monro, D.J. Richardson, U. Keller, Opt. Lett. 28, 1951 (2003)

10. C.J. Saraceno, O.H. Heckl, C.R.E. Baer, T. Sudmeyer, U. Keller, Opt. Express 19, 1395 (2011)

11. O.H. Heckl, C.J. Saraceno, C.R.E. Baer, T. Sudmeyer, Y.Y. Wang, Y. Cheng, F. Benabid, U. Keller, Opt. Express 19, 19142 (2011)

12. F.X. Kärtner, I.D. Jung, U. Keller, IEEE J. Sel. Top. Quantum Electron. 2, 540 (1996)

13. F. Druon, F. Balembois, P. Georges, Ann. Chim. Sci. Mater. 28, 47 (2003)

14. A. Giesen, J. Speiser, R. Peters, C. Krankel, K. Petermann, Photonics Spectra 41, 52 (2007)

15. F.X. Kärtner, U. Keller, Opt. Lett. 20, 16 (1995)

16. R. Paschotta, U. Keller, Appl. Phys. B 73, 653 (2001)

17. J. Aus der Au, G.J. Spühler, T. Südmeyer, R. Paschotta, R. Hövel, M. Moser, S. Erhard, M. Karszewski, A. Giesen, U. Keller, Opt. Lett. 25, 859 (2000)
18. F. Brunner, T. Südmeyer, E. Innerhofer, R. Paschotta, F. MorierGenoud, J. Gao, K. Contag, A. Giesen, V.E. Kisel, V.G. Shcherbitsky, N.V. Kuleshov, U. Keller, Opt. Lett. 27, 1162 (2002)

19. D.E. Spence, P.N. Kean, W. Sibbett, Opt. Lett. 16, 42 (1991)

20. O. Pronin, J. Brons, C. Grasse, V. Pervak, G. Boehm, M.C. Amann, V.L. Kalashnikov, A. Apolonski, F. Krausz, Opt. Lett. 36, $4746(2011)$

21. K. Petermann, G. Huber, L. Fornasiero, S. Kuch, E. Mix, V. Peters, S.A. Basun, J. Lumin. 87-89, 973 (2000)

22. R. Peters, C. Kränkel, S.T. Fredrich-Thornton, K. Beil, K. Petermann, G. Huber, O.H. Heckl, C.R.E. Baer, C.J. Saraceno, T. Sudmeyer, U. Keller, Appl. Phys. B 102, 509 (2011)

23. S. Pekarek, C.J. Saraceno, O.H. Heckl, C.R.E. Baer, C. Schriber, M. Golling, T. Sudmeyer, K. Beil, C. Krankel, G. Huber, U. Keller, Self-referenceable high-power frequency comb from a 7-W, 142fs $\mathrm{Yb}: \mathrm{Lu}_{2} \mathrm{O}_{3}$ thin disk laser oscillator, presented at the Ultrafast Optics, 2011, post-deadline paper PD3

24. K.S. Bagdasarov, A.A. Kaminskii, A.M. Kevorkov, L. Li, A.M. Prokhorov, T.A. Tevosyan, S.E. Sarkisov, Sov. Phys. Dokl. 20, 681 (1975)

25. R. Peters, K. Petermann, G. Huber, in Advanced Solid-State Photonics (ASSP), Denver, USA (2009). Paper MC4

26. C.J. Saraceno, O.H. Heckl, C.R.E. Baer, M. Golling, T. Sudmeyer, C. Krankel, K. Beil, K. Petermann, G. Huber, U. Keller, Opt. Express 19, 20288 (2011)

27. G.B. Venus, A. Sevian, V.I. Smirnov, L.B. Glebov, in Conference on High-Power Diode Laser Technology and Applications III, San Jose, CA (2005), p. 166

28. M. Haiml, R. Grange, U. Keller, Appl. Phys. B 79, 331 (2004)

29. V. Magni, J. Opt. Soc. Am. A 4, 1962 (1987)

30. C. Hönninger, R. Paschotta, F. Morier-Genoud, M. Moser, U. Keller, J. Opt. Soc. Am. B 16, 46 (1999)

31. C.J. Saraceno, C. Schriber, M. Mangold, M. Hoffmann, O.H. Heckl, C.R.E. Baer, M. Golling, T. Sudmeyer, U. Keller, IEEE J. Sel. Top. Quantum Electron. (2012). doi:10.1109/ JSTQE.2010.2092753 